\title{
Type 2 Diabetes Prescribing Habits amongst Providers to an Underserved Population
}

Cynthia A. King, PharmD, BCACP, CACP1; Atem A. Fontem, PharmD ${ }^{2}$; Benjamin S. King, PharmD, BCACP²; David Gothard, PhD ${ }^{3}$

${ }^{1}$ MetroHealth System; Northeast Ohio Medical University; ${ }^{2}$ MetroHealth System; ${ }^{3}$ Data Analysis for Clinical Research Studies

\begin{abstract}
Introduction: Type 2 diabetes mellitus (T2DM) affected over 30 million individuals in the United States as of 2015. Due to the national diabetes guidelines recommending drug selection based on several patient specific factors and varying formulary restrictions, prescribers are often inundated when selecting treatment. Currently, limited evidence is available regarding the primary factors influencing prescribers' drug therapy selection.

Objectives: The purpose of this study was to identify factors that influence providers during T2DM medication selection.

Methods: The study was conducted with providers at a large, academic, safety net health system. All prescribers were sent an electronic, optional and anonymous survey. Prescribers treating T2DM in non-pregnant adult patients were the only prescribers assessed. Factors evaluated were: cost, A1c, comorbidities, adherence, weight, tolerability, patient limitations, and use of guidelines.

Results: A total of 86 prescribers responded, yielding a response rate of $31 \%$. The respondents included physicians (56.3\%), nurse practitioners (21.8\%), medical residents (18.4\%), and fellows (3.4\%); with the majority practicing in internal or family medicine (47.1\%). The most frequently prescribed T2DM medications included: metformin (83.8\%), insulin (78.1\%), and sulfonylureas (64.8\%). Cost and A1c elevation were two of the major factors influencing prescribing of metformin (94.1\% and $81.2 \%)$, insulin (57.4\% and 69.6\%), and sulfonylureas (81.2\% and 89.9\%) respectively. Due to cost concerns, respondents reported rarely or never prescribing glucagon-like peptide-1 agonists (GLP-1RA) and sodium-glucose co-transporter-2 inhibitors (SGLT2i) despite recognizing benefits on diabetes related comorbidities.

Conclusion: Although current literature from the national guidelines encourages the use of GLP-1RA and SGLT2i as first-line options after metformin in T2DM, these classes of medications were not reported among the most commonly prescribed despite providers correctly identifying positive medication attributes such as cardio- and nephroprotection and weight loss. However, cost of these medications appears to outweigh the benefits when selecting medication therapy.
\end{abstract}

Keywords: Type 2 diabetes mellitus, prescribing habits, clinical decision making, comorbidities, treatment choices

\section{Background}

Type 2 diabetes mellitus (T2DM) is a significant health problem in the United States. With 30.3 million diabetic cases, 90-95\% of cases are T2DM with $36.2 \%$ of T2DM patients uncontrolled on their current regimen. ${ }^{1,2}$ The economic impact of T2DM is significant, with the annual cost of diagnosed diabetes in 2017 being $\$ 327$ billion, a combination of $\$ 90$ billion in reduced productivity and $\$ 237$ billion in direct medical costs. ${ }^{3}$ Most T2DM patients have at least one other comorbidity; the most common comorbidities include: hypertension (82.1\%), obese/overweight (78.2\%), chronic kidney disease (CKD) (24.1\%), cardiovascular disease (CVD) (21.6\%), and heart failure (HF) (7.4\%). ${ }^{4}$ The landscape of T2DM management has evolved considerably over the last 15 years with over ten antidiabetic drug classes available as treatment options. In addition to new therapy, national guidelines are frequently updated with a new focus on treatment selection to further manage comorbid conditions. ${ }^{5-7}$ Due to all of these factors, optimal management of T2DM is often challenging and complex.

Corresponding author: Cynthia A. King, PharmD, BCACP, CACP MetroHealth System; Northeast Ohio Medical University Email: cking3@metrohealth.org
Minimal literature exists evaluating how providers weigh patient-specific factors to determine drug therapy selection for the management of T2DM. In 2007, a national survey of physician members of the American Diabetes Association or the Society of General Internal Medicine who managed patients with T2DM sought to identify the major considerations leading to T2DM drug selection. When choosing to initiate drug therapy, the top three most common factors considered were: assessment of the patient's health status and comorbid conditions (89\%), A1c elevation (74\%), and patient's weight $(66 \%) .{ }^{8}$ At the time of this study, national guidelines did not have a strong emphasis of drug selection based on comorbidities. In addition, dipeptidyl peptidase-4 inhibitors (DPP-4i) and glucagon-like peptide-1 receptor agonists (GLP1RA) were new to the market and sodium-glucose cotransporter-2 inhibitors (SGLT2i) were not yet available.

More recently, another study evaluated over one million T2DM patients to assess changes in prescribing patterns from 2005 to 2016. During this time, prescribing patterns for monotherapy antidiabetic regimens showed an increase in metformin use (60\% to $77 \%)$, a decrease in sulfonylurea (SU) (20\% to $8 \%$ ) and thiazolidinedione (TZD) use (11\% to $0.7 \%)$, while insulin use remained consistent ( $8 \%$ to $10 \%$ ). Over the course of the study, prescribing patterns for add-on therapies showed a decrease in SU use (60\% to $48 \%$ ) and an increase in insulin use (7\% to $17 \%)$. Specifically in patients on metformin monotherapy, SU, DPP-4i, 
and insulin were the most commonly prescribed add-on medications. The newer antidiabetic classes, such as DPP-4i (0.4\% to $20 \%$ ), GLP-1RA (3\% to $7 \%$ ), and SGLT2i (0\% to $7 \%$ ) saw increased use; however, GLP-1RA and SGLT2i were suboptimally used. ${ }^{9}$

Limitations in the current literature related to prescribing of diabetic regimens include changes in available of drug classes, knowledge of drug selection to benefit other comorbidities, and changes in recommendations from guidelines. With approximately one in three patients not being adequately controlled on their current diabetes regimen, it is important to understand the existing prescribing habits and clinical decision making to improve patient care and outcomes. Considering this knowledge gap, the purpose of this study was to identify factors that influence providers in a large academic safety net health system, when selecting antidiabetic medications.

\section{METHODS}

\section{Study Design}

Prescribers from a large academic safety net health system located in the Midwest were surveyed on their prescribing habits and reasoning for drug selection for the treatment of T2DM. The health system has 22 community medical facilities with over one million outpatient visits annually serving mainly low-income, underserved patients. The patient population treated by the health system is composed of following patient demographics: insurance type includes $37.6 \%$ with Medicaid, $27.3 \%$ with Medicare, and $11.3 \%$ self-pay; age groups include $35.8 \%$ ages $45-64$ years and $23.2 \%$ ages 65 years and older; and ethnicities include $35.8 \%$ African American and $51.9 \%$ Caucasian. Nearly $20 \%$ of patients seeking treatment within the health system are living at or near the federal poverty line. A total of 275 prescribers were eligible to complete the survey; this included medical residents, medical fellows, physicians, nurse practitioners, and physician assistants. Providers who prescribed medications to treat T2DM in non-pregnant adult patients were included in the study. Providers working in internal or family medicine clinics were considered generalists, while providers working in endocrinology, nephrology, gerontology, infectious disease (HIV), and others, were considered specialists. To minimize the length of time required to complete the survey, drug classes such as alpha-glucosidase inhibitors, amylin analogs, bile acid sequestrants, and meglitinides were excluded, as they are no longer recommended for use in the treatment of T2DM. This study was approved as exempt by the Institutional Review Board.

\section{Survey}

An optional anonymous electronic 17-question survey was built by the research team using the Verient ${ }^{\mathrm{TM}}$ survey software and distributed via email between November 29th, 2018 and December 30th, 2018 with two reminder emails sent to improve the response rate during the one-month survey period. A link in the email directed prescribers to a cover letter describing the study and allowed for prescribers to give consent to participate in the study. Providers were informed that the survey will take approximately 10 to 15 minutes. They were asked to provide demographic information including their professional designation, years of practice, time spent in clinic, practice department, geographical location, average time between follow-up appointments for T2DM patients, reason for this follow-up period, and frequency of time that cost impacted drug therapy selection. Prescribers were asked to rank ("never", "rarely", "often", "very often") how frequently they prescribed a biguanide, DPP-4i, GLP-1RA, SGLT-2i, TZD, SU, and insulin. In order to assess clinical decision making, providers were asked to select "positive", "negative" or "neutral" response for each drug class for each of the following factors: cost/insurance coverage, extent of A1c elevation, patient's comorbidities (cardiovascular/renal benefits), patient's adherence (frequency/route of administration), patient's weight/BMI, patient's tolerability (side effects/adverse drug reactions), social/physical/mental limitations, and use of guidelines/consensus statements. If the provider selected "positive", this means that the drug is considered beneficial to most patients, and vice versa for a "negative" response. For each of the clinical decision-making criteria, prescribers were provided a definition of a positive and a negative response. For example, the perception of cost was described a positive if the medication was affordable to patients and did not require prior authorizations versus negative if the medication was cost prohibitive, required prior authorization or was not covered by insurances (Appendix 1).

\section{OUTCOMES ANALYSIS}

The primary objective was to assess the positive, neutral, or negative perception of each clinical decision-making criteria to the respective drug class. The secondary objectives included describing the frequency of prescribing type 2 diabetes medication classes by providers, comparing the differences in clinical decision making between specialists and generalists, comparing the differences in clinical decision making between providers based on years of practice and comparing the differences in clinical decision making between providers based on geographical location.

\section{DATA ANALYSIS}

Survey data were imported into SPSSv25.0 (IMB Corp.) software for analysis. Categorical data were summarized using frequencies and percentages. Comparison for distributional equality were performed using Pearson chi-squared tests or Fisher's exact test depending on cell sample sizes. Post-hoc Bonferroni adjusted z-tests were used to detect specific distributional differences in the presence of overall distributional inequality. Continuous data were summarized using means and standard deviations and analyzed for between group mean equality using Student's t-tests. Unless otherwise indicated all statistical testing was two-sided with alpha set at 0.05 . 


\section{RESULTS}

A total of 86 providers participated in the survey, yielding a response rate of $31 \%$ (Table 1 ). The geographical distributions of all our respondents were unevenly balanced, with $46.3 \%$ of providers being from one location. Based on demographic data, the responding providers have been practicing for an average of 13.85 years and see patients in the office/clinic an average of three eight-hour days per week (full time equivalent of 0.58 ).

Amongst the seven medication classes, metformin (85\%), SU $(78 \%)$, and insulin $(66 \%)$ were prescribed most frequently, when combining both "often" and "very often" responses. Four drug classes were prescribed infrequently (identified as 'rarely' or 'never' on the survey) included 95.9\% (TZD), 91.6\% (SGLT2i), $76.8 \%$ (GLP-1RA), and $57.6 \%$ (DPP-4i) of the time (Figure 1). The most common clinical decision-making factors considered when prescribing the most frequently prescribed classes were cost and extent of A1c elevation. The selection of metformin, insulin, and SU seemed to be largely driven by cost $(94.1 \%$, $56.7 \%$, and $80.9 \%$ respectively), and extent of A1c elevation $(80.9 \%, 89.7 \%$, and $69.1 \%$ respectively). Also, use of guidelines/consensus statements was the third reason for selection of metformin and insulin (87.3\% and $76.2 \%)$, versus patients' adherence, which was the third reason for SU selection (64.7\%) (Table 2).

Some differences noted amongst provider prescribing were that specialists identified more positive benefits for SGLT2i ( $p=$ 0.011 ), while generalists identified more positive benefits for SU $(p=0.001)$. There were no differences seen between providers based on years of practice. There were also no differences seen based on follow-up appointment time frame and drug selection. Geographical location had no impact on drug selection either when compared across all locations or when compared at locations with and without the ambulatory care pharmacists.

\section{DISCUSSION}

The findings of this study show the complexity involved when prescribing drug therapy for T2DM management. This study explored most of the key clinical factors recommended by the national guidelines. The prescribing habits of the providers could be influenced by the ambulatory care pharmacy specialists who are being referred approximately one thousand patients every year; however, this did not seem to be a significant impact based on the statistical analysis.

The use of metformin is very consistent with guideline recommendations, and this accentuates the fact the providers have an appropriate understanding and utilization of this firstline medication. The most frequently prescribed medication classes (metformin, SU, insulin) were highly viewed as cost effective likely due to all these drug classes being represented on the 340B formulary utilized at the on-site pharmacies within the health system. The importance of cost is known to be a major factor nationwide; however it may have been over emphasized by providers due to the underserved patient population to which they provide care. ${ }^{9}$ It is important to note that while GLP-1RA and SGLT2i were not frequently prescribed likely due to a negative association with cost, the drug classes were correctly identified as beneficial to patient comorbidities. As nearly $25 \%$ of T2DM patients have CKD, $20 \%$ have CVD and $8 \%$ have heart failure, utilization of T2DM medications to also benefit these comorbidities is important extremely important when selecting therapy for the management of T2DM. ${ }^{4}$ Based on the data from this study, further provider education is needed to make providers aware of formulary inclusions for Medicaid patients, copay cards for commercial patients, and formulary availability of $340 \mathrm{~b}$ medications that often make brand name medications significantly more affordable for patients.

This study provides novel insight into the prescribing patterns and rationale for antidiabetic regimens. Compared to prior studies, the surveyed prescribers have increased exposure as all current antidiabetic medication classes have been on the market for at least five years. Also, the recent transition of T2DM guidelines to an increased focus on pharmacologic intervention based on patient comorbidities and concerns can also change prescribing habits. Although the risk of bias was minimized, limitations related to recall bias were possible as prescribers may recall primarily their most recent interventions. Selection bias is also possible due to a lack of respondents from physician assistants and all respondents originating from a single health system. Convenience of the survey and short survey length most likely contributed to the high response rate. Online surveys of providers have a response rate of $11 \%$ to $39 \%$ with the majority response rates being less than $25 \%{ }^{10-12}$ While the short survey length may have increased response rate, minimizing length came at the cost of the ability to capture interclass variabilities and assessment of other patient factors such as hypoglycemia.

\section{CONCLUSION}

In summary, the most frequently prescribed T2DM medication classes at this large academic safety-net hospital were metformin, SU, insulin. Cost was a significant driving factor in drug selection in the management of T2DM. Based on these results, additional provider education should be provided regarding updates on national guideline recommendations, changes in insurance formularies, and target patient populations (comorbidities, weight, hypoglycemia risks) where drug selection may differ. The results of this study should be interpreted in the context of the study design and patient population/healthcare system, as they may not be generalizable to other health care systems.

Acknowledgements: none

Conflict of Interest: The authors declare no conflicts of interest. 


\section{References}

1. Centers for Disease Control and Prevention. "National diabetes statistics report, 2017." Atlanta, GA: Centers for Disease Control and Prevention. 2017.

2. Carls G, Huynh J, Tuttle E, et al,. "Achievement of glycated hemoglobin goals in the US remains unchanged through 2014." Diabetes Therapy. 2017; 8(4): 863-873.

3. American Diabetes Association. Economic costs of diabetes in the U.S. in 2017. Diabetes Care. 2018; 41(1): 917-928.

4. Iglay K, Hannachi $\mathrm{H}$, Joseph Howie $\mathrm{P}$, et al,. "Prevalence and co-prevalence of comorbidities among patients with type 2 diabetes mellitus." Current Medical Research and Opinion. 2016;32(7):1243-52.

5. Cavaiola T, Pettus J. "Management of type 2 diabetes: selecting amongst available pharmacological agents." MDText. 2017.

6. American Diabetes Association. "Standards of medical care in diabetes-2019." Diabetes 2019; 42(1): S1S193.

7. Garber A, Abrahamson M, Barzilay J, et al,. "Consensus statement by the American Association of Clinical Endocrinologists and American College of Endocrinology on the comprehensive type 2 diabetes management algorithm-2018 executive summary." Endocrine Practice. 2018; 21(4): 91-120.

8. Grant R, Wexler D, Watson A, et al,. "How doctors choose medications to treat type 2 diabetes: a national survey of specialists and academic generalists." Diabetes Care. 2007; 30(6): 1448-1453.

9. Montvida O, Shaw J, Atherton J, et al,. "Long-term trends in antidiabetes drug usage in the US: realworld evidence in patients newly diagnosed with type 2 diabetes." Diabetes care. 2018; 41(1): 69-78.

10. Aerny-Perreten N, Dominquez-Berjon MF, EstebanVasallo MD, et al. "Participation and factors associated with late or non-response to an online survey in primary care." J Eval Clin Pract. 2015;21(4):688-693.

11. Scott $\mathrm{A}$, Jeon $\mathrm{SH}$, Joyce $\mathrm{CM}$, et al. "A randomized trial and economic evaluatin of the effect of response mode on response rate, response bias, and item nonresponse in a survey of doctors." BMC Med Res Methodol. 2011:11:126.

12. Cook CA, Wittich CM, Daniels WL, et al. "Incentive and reminder strategies to improve response rate for internet-based physician surveys: a randomized experiment." J Med Internet Res. 2016;18(9):e244. 
Table 1: Baseline characteristics

Demographic data for survey participants.

\begin{tabular}{|r|c|}
\hline \multicolumn{1}{|l|}{ Demographics } & Number (\%) \\
\hline Professional Designation & \\
\hline Physician & $48(55.8)$ \\
\hline Nurse Practitioner & $19(22.1)$ \\
\hline Resident & $16(18.6)$ \\
\hline Fellow & $3(3.5)$ \\
\hline Practice & \\
\hline Generalists & $57(66.3)$ \\
\hline Specialist & $29(33.7)$ \\
\hline
\end{tabular}

Table 2: Clinical Decision-Making Rationale

Includes the major considerations respondents considered when deciding to prescribe or not prescribe certain T2DM drug classes.

\begin{tabular}{|c|c|c|c|c|c|c|c|c|c|c|c|c|c|c|}
\hline \multirow[t]{2}{*}{ Clinical decision-making (\%) } & \multicolumn{2}{|c|}{ Biguanides } & \multicolumn{2}{|c|}{ DPP-4i } & \multicolumn{2}{|c|}{ GLP-1RA } & \multicolumn{2}{|c|}{ Insulin } & \multicolumn{2}{|c|}{ SGLT2i } & \multicolumn{2}{|c|}{ SU } & \multicolumn{2}{|c|}{ TZD } \\
\hline & POS & NEG & POS & NEG & POS & NEG & POS & NEG & POS & NEG & POS & NEG & POS & NEG \\
\hline Cost & 94.1 & 0 & 20.6 & 38.2 & 8.8 & 64.7 & 56.7 & 9 & 6 & 65.7 & 80.9 & 1.5 & 22.7 & 16.7 \\
\hline Extent of A1c elevation & 80.9 & 1.5 & 55.9 & 4.4 & 68.2 & 3 & 89.7 & 0 & 33.3 & 6.1 & 69.1 & 4.4 & 22.4 & 11.9 \\
\hline Other comorbidities & 73.5 & 4.4 & 27.9 & 1.5 & 57.4 & 1.5 & 42.6 & 10.3 & 44.8 & 9 & 20.9 & 16.4 & 11.8 & 41.2 \\
\hline Guideline recommendations & 87.3 & 0 & 43.3 & 1.7 & 50 & 4.8 & 76.2 & 0 & 32.2 & 11.3 & 46 & 11.1 & 13.1 & 36.1 \\
\hline Weight/BMI & 71.9 & 4.7 & 15.2 & 4.7 & 65.6 & 1.6 & 7.9 & 68.3 & 21.9 & 4.7 & 7.8 & 48.4 & 0 & 41.3 \\
\hline Adherence & 63.2 & 5.9 & 66.2 & 4.4 & 43.3 & 13.4 & 23.5 & 36.8 & 32.8 & 6 & 64.7 & 1.5 & 37.9 & 6.1 \\
\hline Limitations & 68.3 & 1.6 & 43.5 & 8.1 & 4.9 & 36.1 & 9.7 & 54.8 & 22.6 & 9.7 & 49.2 & 0 & 35.5 & 6.5 \\
\hline Patient's preference & 33.3 & 23.8 & 50.8 & 0 & 25.8 & 4.8 & 34.9 & 14.3 & 14.3 & 15.9 & 30.2 & 12.7 & 11.3 & 40.3 \\
\hline
\end{tabular}

POS = positive; NEG = Negative 


\section{Figure 1: Frequency of Prescribed Medications}

Includes the frequency at which certain T2DM drug classes where prescribed at the large, academic, safety net health system in the Midwest. Ranging from "never" to "very often".

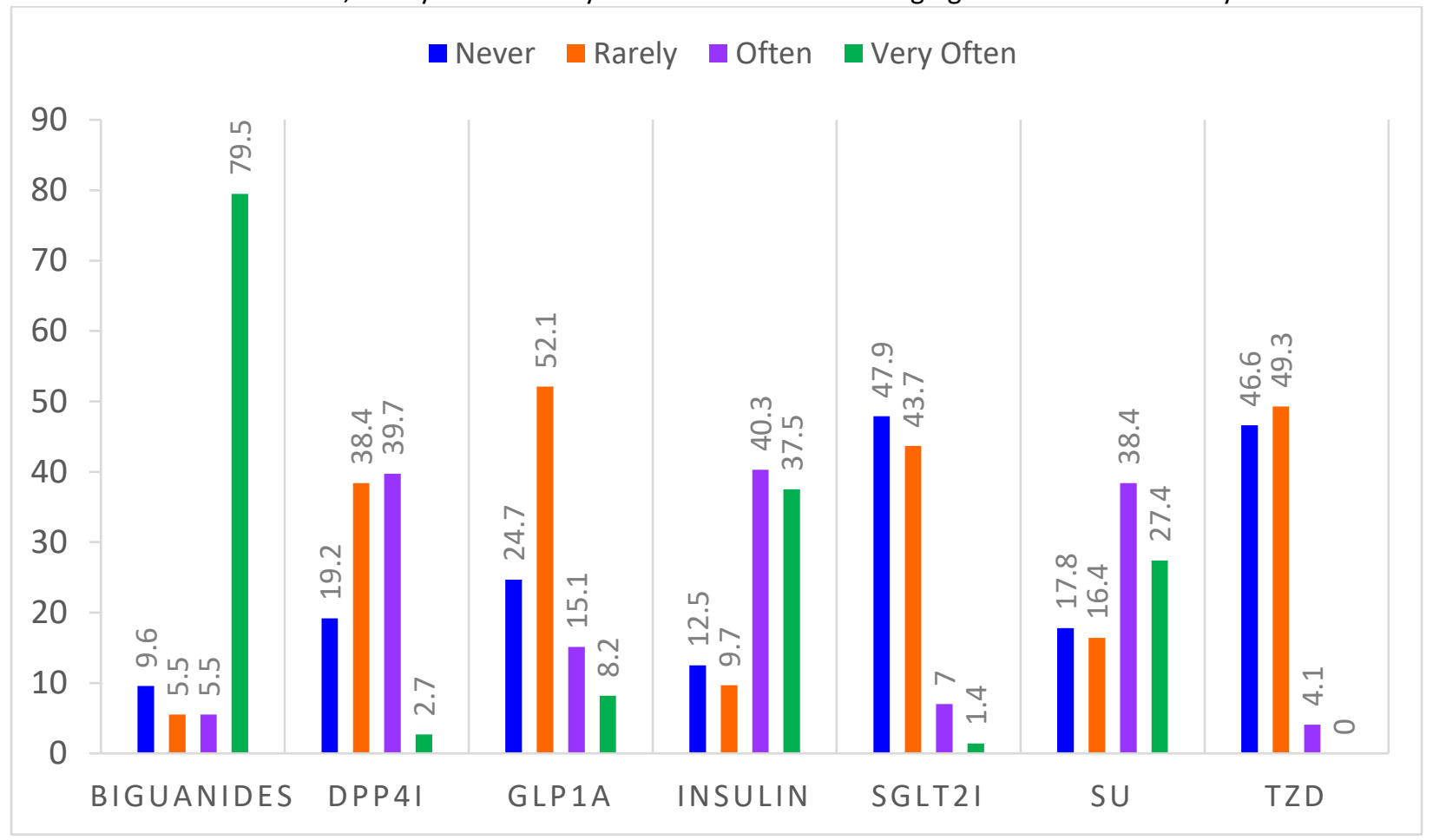


Appendix 1: Survey questionnaire

The survey questionnaire provided to medical residents, fellows, nurse practitioners, physician assistants, and physicians concerning the prescribing of diabetes meditations in the management of T2DM.

1. Do you prescribe medications to treat type 2 diabetes in non-pregnant, adult patients in the outpatient setting?
a. Yes
b. No

2A. Please select your professional designation.
a. Fellow
b. Resident
c. Certified Nurse Practitioner
d. Physician Assistant
e. Physician (MD/DO)

2B. Please select your area of specialty
a. Internal Medicine or Family Medicine
b. Endocrinology
c. Geriatrics
d. Other

3. How many years have you been in practice? (Round to the nearest full year)

4. Which of the following site is your main practice site ( $>50 \%$ of patient care time)

5. What part of an FTE do you spent on direct patient care (0.2FTE $=8$ hour day)? (response 0.1-1 FTE)

6. How often does cost of the antidiabetic medications, whether due to insurance copay, gap in Medicare coverage, or lack of insurance, prevent you from prescribing your preferred Type 2 diabetic regimen for your patients?
a. Less than $25 \%$
b. $25-50 \%$
c. $50-75 \%$
d. Greater than $75 \%$

7. On average, how often do you see uncontrolled T2DM patients for follow up?
e. < 1 month
f. 1-2 months
g. 3-4 months
h. 5-6 months
i. $>6$ months

8. What is the main driving force for this follow-up time frame?
j. Medication titration
k. Timing of next $\mathrm{HbA1c}$
I. Patient request
m. Availability of provider schedule
n. Assessment of medication change or patient status

9. How often do you prescribe the following medications in your daily practice? Please select appropriate response for each drug class. Multiple Choice (never, rarely, often, very often).

*The survey provided through the survey software, after each drug class, the brand and generic names were listed to improve correct identification/recognition.

\begin{tabular}{|c|l|l|l|l|}
\hline Drug Class & Never & Rarely & Often & Very Often \\
\hline Biguanides & & & & \\
\hline DPP4i & & & & \\
\hline GLP1a & & & & \\
\hline Insulin & & & & \\
\hline SGLT2i & & & & \\
\hline SU & & & & \\
\hline TZD & & & & \\
\hline
\end{tabular}


10. How do you perceive each medication class as it relates to each category? Please select positive, neutral, or negative for each category. For example, positively under medication cost means you think the medication is affordable to most patients.

\begin{tabular}{|l|l|l|l|l|l|l|l|}
\hline & Biguanides & DPP4i & $\begin{array}{l}\text { GLP1 } \\
\text { a }\end{array}$ & Insulin & $\begin{array}{l}\text { SGLT2 } \\
\text { i }\end{array}$ & SU & TZD \\
\hline Cost/Insurance & & & & & & & \\
\hline Current A1c & & & & & & & \\
\hline Comorbidity benefit & & & & & & & \\
\hline Adherence (frequency, route, etc.) & & & & & & & \\
\hline $\begin{array}{l}\text { Guidelines/ Consensus statements/ } \\
\text { Usual practice }\end{array}$ & & & & & & & \\
\hline Current weight/ BMI & & & & & & & \\
\hline Side effects/ Adverse Effects & & & & & & & \\
\hline Social, physical, or mental limitations & & & & & & & \\
\hline
\end{tabular}

*The survey provided through the survey software, after each drug class, the brand and generic names were listed to improve correct identification/recognition. On the survey provided, each category was separated into a different question. Consolidated for space considerations. 